\title{
Faddeev Medal
}

Recently, the Topical Group of Few-Body Systems of the American Physical Society and the European Research Committee on Few Body Problems in Physics have announced a new award to recognize distinguished achievement in Few-Body Physics, the Faddeev medal, named in honor of Ludwig Faddeev.

The establishment of this award shows the worldwide recognition of his outstanding contribution to this field of science.

We send our warmest greetings to our Editor-in-Chief L. D. Faddeev on this occasion.

Editorial Board 